\title{
因HAD
}

ISSN-L: 2530-5115

DOI: http://doi.org/10.22585/hospdomic.vli3.10

\section{La privación de libertad en la Hospitalización Terapéutica Obligatoria}

Deprivation of liberty in detention for mandatory hospitalization

Enrique Velez Bueno

Hospital de San Juan de Alicante, Sant Joan d'Alacant, España

Correspondencia/Correspondence

velez_enr@gva.es

Recibido/Received

09.02.2017

Aceptado/Accepted

23.03.2017
Conflicto de Intereses/Competing interest

El autor declara la no existencia de conflicto de intereses de tipo económico ni de cualquier otro tipo.

Velez Bueno E. La privación de libertad en la Hospitalización Terapéutica Obligatoria. Hosp Domic. 2017;1(3): 167-78. 


\section{RESUMEN}

Las personas afectadas por enfermedades infecciosas transmisibles, especialmente tuberculosis, en caso de mostrarse rebeldes al tratamiento correspondiente pueden constituir un grave riesgo para la salud pública. Nuestro ordenamiento jurídico nos proporciona medios para afrontar dichos riesgos principalmente a través de la Ley General de Sanidad y la Ley de Medidas Especiales en Materia de Salud Pública; sin embargo acciones tales como el internamiento involuntario de personas enfermas o infectadas suponen un conflicto con el Derecho a la Libertad de estas por lo que examinaremos cuales son esas medidas y si atentan, efectivamente, contra sus derechos.

Palabras clave: Salud Pública; Derechos Humanos; Aislamiento de Pacientes; Ley General de Sanidad; Hospitalización Terapéutica Obligatoria.

\section{ABSTRACT}

Individuals affected by communicable infectious diseases, specially tuberculosis, if they are refractory to the treatment may constitute a serious risk to public health. Our legal system provides us with tools to face these risks mainly through the Ley de General de Sanidad y Ley de Medidas Especiales en Materia de Salud Pública; however, actions such as the involuntary hospitalization of sick or infected people are in conflict wiht their right to freedom so we'll examine what these measures are and if they actually violate their rights.

Keywords: Public Health; Human Rights; Patient Isolation; Public Health Act; Mandatory Hospitalization. 


\section{INTRODUCCIÓN}

La facilidad de desplazamiento entre los distintos países del mundo lleva consigo, entre otros fenómenos y realidades, la transmisión de patologías y la consiguiente dificultada para controlarlas, lo que ha provocado un repunte de enfermedades infecciosas tales como la tuberculosis. Aunque su tratamiento resulta relativamente fácil en comparación con otras épocas, no obstante, la dificultad surge cuando el paciente se resiste a adoptar éste y las medidas necesarias para el control de su enfermedad. En ese caso concreto, surge un conflicto entre las libertades individuales del paciente y el derecho colectivo a la salud pública pudiendo darse, incluso, la circunstancia de ser necesario el confinamiento forzoso del mismo con objeto de impedir el contagio y obligarlo así a seguir el tratamiento.

La normativa que ampara la adopción de estas medidas se recoge, entre otras normas, en la Ley General de Sanidad y la Ley Orgánica de Medidas Especiales en Materia de Salud Pública. Por otro lado, la libertad es un valor protegido por nuestro Ordenamiento Jurídico por encima de la seguridad, lo que tiene su reflejo en nuestra Constitución y también está protegido por la normativa contenida en los convenios internacionales de los que España forma parte, como la Convención Europea de Derechos Humanos, lo que deja un margen muy estrecho para la aplicación de las disposiciones en materia sanitaria.

\section{LA SALVAGUARDA DE LOS DERECHOS FUNDAMENTALES}

El art. 17 de la Constitución Española, encuadrado en la Sección Primera del Capítulo II del Título I (de los derechos fundamentales y de las libertades públicas), establece lo siguiente:

1. Toda persona tiene derecho a la libertad y a la seguridad. Nadie puede ser privado de su libertad, sino con la observancia de lo establecido en este artículo y en los casos y en la forma previstos en la ley.

En su desarrollo, el resto del artículo se centra en el supuesto de la detención preventiva, si bien el Tribunal Constitucional sentó la doctrina de que la privación de libertad aquí aludida no es exclusivamente la que tiene lugar la que tiene lugar como consecuencia de la comisión de un hecho delictivo (STC. 178/1985). Posteriormente, el art. 24 recoge el derecho a la tutela efectiva de los jueces y tribunales, así como los derechos al Juez ordinario predeterminado por la ley, a la defensa y a la asistencia de letrado, a un proceso público sin dilaciones indebidas y con todas las garantías, a utilizar los medios de prueba pertinentes para su defensa, entre otros.

Por otro lado, el art. 19 CE. recoge el derecho de los españoles a circular por el territorio nacional así como a entrar y salir libremente de España.

En nuestro ordenamiento jurídico son escasas las normas que regulan la limitación de derechos fundamentales por razones sanitarias; aparte de la Ley Orgánica reguladora de los estados de alarma, excepción y sitio (1), podemos mencionar la Ley Orgánica de Medidas Especiales en Materia de Salud Pública (2), publicada al mismo tiempo que la Ley General de Sanidad.

Nuestra Constitución, en el Capítulo quinto del mismo Título I, que trata de la suspensión de los derechos y libertades, en su art. 55, establece dos supuestos de restricciones referentes a los derechos recogidos en los arts. 17 (libertad y seguridad) y 19 (libertad de circulación) que son los casos de suspensión de derechos fundamentales: estados de excepción y sitio, que tienen una di- 
mensión colectiva (art. 55.1) y la suspensión individual en casos de actuación de bandas armadas o elementos terroristas (art. 55.2).

Por aplicación del art. 81 CE. las normas que regulen estas materias han de tener carácter de Ley Orgánica.

Art. 81: «Son leyes orgánicas las relativas al desarrollo de los derechos fundamentales y de las libertades públicas, las que aprueben los Estatutos de Autonomía y el régimen electoral general y las demás previstas en la Constitución.»

Y efectivamente, la Ley de Medidas Especiales en Materia de Salud Pública (como la Ley Orgánica reguladora de los estados de alarma, excepción y sitio) detenta esa naturaleza de ley orgánica pero adolece de cierta ambigüedad y falta de desarrollo que hace difícil su aplicación. Téngase en cuenta que no es posible admitir una interpretación extensiva de la Ley de Medidas Especiales en Materia de Salud Pública porque el art. 4.2 del Código Civil lo impide:

Las leyes penales, las excepcionales y las de ámbito temporal no se aplicarán a supuestos ni en momentos distintos de los comprendidos expresamente en ellas.

Véase como se acordó la segregación de los arts. 21, 22 y 29 del proyecto de Ley General de Sanidad a fin de formar el proyecto de Ley Orgánica sobre Medidas especiales en materia de Salud Pública al entenderse que dicha materia no podía estar regulada por ley ordinaria al afectar a derechos fundamentales.

De hecho, en el debate parlamentario se expresó cierto recelo por algunos diputados a la adopción de medidas de tal trascendencia por parte de la autoridad sanitaria sin supervisión de la autoridad judicial, cuestión a la que luego hemos de aludir (3).

No obstante, recurriendo al mencionado expediente de la Ley Orgánica que prevé el art. 55 CE. se podría llegar al mismo resultado, puesto que era posible declarar mediante decreto acordado en Consejo de Ministros el estado de alarma en caso de crisis sanitarias (tales como epidemias, véase el art. 4.b de la Ley reguladora de los estados de alarma, excepción y sitio). Esto posibilitaría la adopción de las medidas «establecidas en las normas para la lucha contra las enfermedades infecciosas», (art. 12.1) esto es, la adopción de disposiciones recogidas en leyes no orgánicas.

Otra cuestión sería la determinación de las competencias en cuanto al establecimiento de la normativa básica, del desarrollo legislativo y de la ejecución. En este tema está comprometido el derecho a la salud (art. 43 CE.), habiéndose transferido las competencias sanitarias a las comunidades autónomas. Además están en juego los derechos a la libertad y seguridad y a la libertad de circulación (17 y 19 CE.), que son derechos fundamentales. También hay que tener en cuenta las materias a las que hacen referencia los artículos 149.1 .1 (condiciones básicas que garanticen la igualdad de todos los españoles en el ejercicio de los derechos y el cumplimiento de los deberes constitucionales), 149.1.16 (coordinación general de la sanidad), 149.1.18 (procedimiento administrativo común) y 149.1.6 (legislación penal, procesal y penitenciaria) todas ellas de competencia exclusiva del estado.

\section{LA NATURALEZA DE LAS MEDIDAS A ADOPTAR: LA VIIA ADMINISTRATIVA}

Las actuaciones encaminadas al control de la enfermedad corresponden sin lugar a dudas, a la administración sanitaria sin que sea factible en nuestra opinión ningún otro planteamiento. No obs- 
tante, resulta interesante mencionar otros supuestos como la actuación de la jurisdicción penal en determinados casos en que pueda concurrir la comisión de un delito; y también el del internamiento del presunto incapaz, basado en un supuesto de hecho radicalmente distinto, pero que puede presentar analogías procedimentales con el tema a estudio.

Para determinar la posibilidad de actuación de la jurisdicción penal habría que distinguir dos supuestos posibles como son el de que exista una situación de peligro para la salud pública por causa del padecimiento de una enfermedad, pero en la cual la voluntad del enfermo no habría tenido intervención en cuanto a la producción del riesgo o del contagio; y, en segundo lugar, el supuesto de actos en que con intervención de la voluntad del sujeto agente en cualquiera de sus formas, dolosa o imprudente, se produzca un concreto peligro para personas concretas o para la población general.

En el primer caso sólo tendría cabida la actuación de la administración sanitaria, mientras que en el segundo habría una posibilidad de actuación de la jurisdicción penal, cuestión a la que más adelante aludiremos. El enfoque administrativo posibilitaría la aplicación del art. 104 de la Ley 39/2015, de 1 de octubre, del Procedimiento Administrativo Común de las Administraciones Públicas (en adelante LPAC), que establece dentro de los medios de ejecución forzosa de las resoluciones administrativas la compulsión sobre las personas, estableciendo en su párrafo primero:

Los actos administrativos que impongan una obligación personalísima de no hacer o soportar podrán ser ejecutados por compulsión directa sobre las personas en los casos en que la Ley expresamente lo autorice, y dentro siempre del respeto debido a su dignidad y a los derechos reconocidos en la Constitución.

Pero esta medida es un medio de ejecución forzosa por parte de una Administración Pública de una resolución administrativa, por lo que podemos plantearnos la exigencia de un procedimiento dirigido a la producción de la misma resolución, la cual ostentaría el carácter de título.

Así, el art. 97de la LPAC. establece:

1. Las Administraciones públicas no iniciarán ninguna actuación material de ejecución de resoluciones que limite derechos de los particulares sin que previamente haya sido adoptada la resolución que le sirva de fundamento jurídico.

2. EL órgano que ordene un acto de ejecución material de resoluciones estará obligado a notificar al particular interesado la resolución que autorice la actuación administrativa.

Otra opción es la de prescindir del procedimiento administrativo. Eduardo García de Enterría y Tomás-Ramón Fernández (4) distinguen el caso de la actuación en virtud de una simple «orden de ejecución» de aquel otro en que la orden tenga su origen en un título ejecutivo que dé lugar a los resortes de la ejecución forzosa, contraponiendo así la «coacción directa» frente a la ejecución forzosa. A efectos prácticos la primera se diferenciaría de la segunda en que sólo en esta última existe una previa notificación al interesado, con la consiguiente posibilidad de cumplimiento voluntario.

Puede parecer un sistema mucho más ágil y apropiado a los fines examinados, aunque también hay que tener en cuenta el privilegio de la ejecutoriedad inmediata de los actos administrativos y su mantenimiento no obstante su eventual impugnación administrativa o jurisdiccional (arts. 38, 98 y 117 de la LPAC) De esta forma las medidas dictadas por la correspondiente administración se estarían cumpliendo desde un principio, no obstante la progresión de un procedimiento administrativo.

De cualquier forma, ha de existir un acto administrativo previo, revisable en vía ContenciosoAdministrativa, conforme al art. 97.2 de la LPAC. antes visto, si bien la Ley Reguladora de la Jurisdicción Contencioso Administrativa también permite acudir a la vía judicial en caso de actuaciones de hecho. 
En la práctica, el legislador ha optado por establecer en el art. 9 de la Ley básica reguladora de la autonomía del paciente lo siguiente (5):

Los facultativos podrán llevar a cabo las intervenciones clínicas indispensables en favor de la salud del paciente, sin necesidad de contar con su consentimiento, en los siguientes casos:

- a) Cuando existe riesgo para la salud pública a causa de razones sanitarias establecidas por la Ley. En todo caso, una vez adoptadas las medidas pertinentes, de conformidad con lo establecido en la Ley Orgánica 3/1986, se comunicarán a la autoridad judicial en el plazo máximo de 24 horas siempre que dispongan el internamiento obligatorio de personas.

(...)

Dicha norma parece prescindir de todo procedimiento en vía administrativa para decantarse por una supervisión judicial, aparte del control de la Jurisdicción Contencioso-Administrativa.

\section{LA SENTENCIA DEL TSJCM 48/2001 (6)}

Es escasa la aplicación de las medidas previstas en la LO 3/86, aparte de la falta de desarroIlo normativo de la misma y, en consecuencia, poca la jurisprudencia producida al respecto. La sentencia 48/2001 del Tribunal Superior de Justicia de la Comunidad de Madrid (TSJCM), previo reconocimiento del vacío legal existente en esta materia, recurre resueltamente al expediente de la analogía como método de integración de esta laguna legal aplicando al afectado por la Hospitalización Terapéutica Obligatoria las mismas garantías que recoge el art. 763 Lec. para el internamiento no voluntario por razón de trastorno psíquico.

Así, en su fundamento de derecho segundo se recoge lo siguiente:

«Nos encontramos en el caso presente y en los supuestos que hipotéticamente pudieran presentarse en lo sucesivo, con un vacío legal de carácter procedimental que resulta especialmente grave y preocupante por lo que pudiera implicar de colisión entre derechos constitucionalmente protegidos que pudieran entrar en conflicto y cuya salvaguardia viene encomendada «ex» art. $124 \mathrm{CE}$ al Ministerio Fiscal.

Dado este vacío legal ante un supuesto de limitación o privación del derecho a la libertad de una persona y tratando de efectuar una labor integradora en la aplicación de la norma jurídica, parece a este Ministerio que el supuesto más próximo y cercano sería el contemplado en la LECiv, art. 763, referido al internamiento no voluntario por razón de trastorno psíquico, pese a constatar la diferencia consistente en el defecto de capacidad presente en el enajenado y ausente en supuestos como el que nos ocupa.

Sentado lo anterior parece que la situación jurídica del que padece una enfermedad física contagiosa potencialmente peligrosa para la colectividad no ha de ser de peor condición, dando por hecho la necesidad de internamiento, que la del enfermo mental objeto de internamiento no voluntario, al que no se olvide se otorga control jurisdiccional aunque su capacidad esté suplida o complementada por padre o tutor.»

La revisión que realiza de la sentencia del Juzgado de lo Contencioso-Administrativo de Madrid es totalmente garantista de los derechos de la persona afectada por la hospitalización, estableciendo un exhaustivo examen de las medidas a adoptar en defensa de los mismos en el mismo fundamento de derecho segundo: 
«1. Audiencia del interesado, persona que, no se olvide, es plenamente capaz, dada su situación física y no mental, sin perjuicio de la lógica e indispensable adopción de cuantas medidas profilácticas se estimen oportunas.

2. Ofrecimiento de la posibilidad que le asiste de designar Letrado y Procurador dado que la intervención del Fiscal, sin perjuicio de su actuación institucional, no se refiere exclusivamente a la defensa del interesado, sino que paralelamente tiene también atribuida la defensa de la salud pública de los ciudadanos por aplicación del art. 124 CE en relación con el art. 15 CE, intereses que pueden resultar contrapuestos.

3. Control de la persistencia en el tiempo de las circunstancias habilitantes y de su materialización, dado que no parece respetuoso con el derecho del art. 17 CE adoptar una medida de internamiento en cualquier lugar "en que se considere oportuno" y sin duración máxima ni fijación de períodos razonables de revisión.

Por ello entiende el Fiscal que la autorización no debe amparar el cumplimiento de la medida en cualquier lugar que no sea puntualmente conocido por el Juzgado, de tal forma que si la Administración Sanitaria a quien corresponde determinar dicho lugar considerara que procede el traslado a otro centro diferente del Instituto Carlos III comunicarlo inmediatamente al Juzgado, que sigue teniendo las facultades de control de la medida.

4. Respecto del tiempo, el plazo de nueve meses a que también alude la Clínica Médico Forense, no puede ser inicialmente un plazo mínimo, sino máximo, sin perjuicio, claro está, de cuantas prórrogas fueran necesarias, ya que parece incompatible con el art. 17 CE la fijación de mínimos y no de máximos.»

\section{LAS MEDIDAS ESPECIALES EN LA NORMATIVA VALENCIANA}

La Ley de Salud de la Comunidad Valenciana (7) que deroga a la anterior Ley de Salud Pública de la Comunidad Valenciana (8) desarrolla una serie de medidas en su título VIII, de la Intervención y medidas especiales, dividido en dos Capítulos, sobre la Autoridad pública sanitaria y la intervención en materia de sanidad y sobre las medidas especiales cautelares y definitivas.

En el segundo capítulo se incluye:

«Artículo 86 Medidas especiales cautelares y definitivas.

1. Cuando la actividad desarrollada pudiera tener una repercusión excepcional y negativa en la salud de los ciudadanos, las autoridades públicas sanitarias, a través de sus órganos competentes en cada caso, podrán proceder a la adopción de las medidas especiales que resulten necesarias para garantizar la salud y seguridad de los ciudadanos, que tendrán carácter cautelar $\mathrm{O}$, tras el correspondiente procedimiento contradictorio, carácter definitivo.»

Y sus párrafos tercero y cuarto establecen que la duración de las medidas de carácter temporal a que se refieren los apartados anteriores no excederá de lo que exija la situación que la motiva, ello sin perjuicio de las posibles prórrogas que puedan acordarse mediante resolución motivada y que las medidas cautelares deberán ser confirmadas, modificadas o levantadas en el acuerdo de iniciación del procedimiento, que deberá efectuarse dentro de los quince días siguientes a su adopción.

Posteriormente el artículo 89.2 referido a la intervención de medios personales establece lo siguiente:

"Cuando exista o se sospeche razonablemente la existencia de un riesgo inminente y extraordinario para la salud de la población debido a la situación sanitaria concreta 
de una persona o grupos de personas, se podrá ordenar la adopción de las medidas preventivas generales y de intervención entre las que se incluyen las de reconocimiento, tratamiento, hospitalización o control individual sobre la persona o grupos de personas, mediante decisión motivada, por el tiempo necesario para la desaparición del riesgo. La adopción de medidas que impliquen privación o restricción de la libertad o de otro derecho fundamental está sujeta a lo dispuesto en la legislación estatal vigente en materia de medidas especiales en salud pública y la reguladora de la jurisdicción contencioso-administrativa.»

Por último, el artículo 91 establece que el plazo para resolver y notificar en los procedimientos administrativos de adopción de medidas especiales será de seis meses contados desde la fecha en que se dicte el acuerdo de incoación.

Respecto de la duración de estas medidas, según el artículo 86.3 antes visto establecía, no excederá de lo que exija la situación que la motiva, aunque permite sucesivas prórrogas, siempre acordadas mediante resolución motivada. De esta forma no se establece ningún plazo concreto, sino que se deja a criterio de los profesionales y autoridades competentes. Esta indefinición no existe, por ejemplo, en el artículo 763 de la Ley de Enjuiciamiento Civil, el cual regula el internamiento no voluntario por razón de trastorno psíquico; en el mismo se establece muy claramente la distribución en el tiempo de las diferentes etapas del proceso, lo que no impide que la medida se prolongue durante todo el tiempo en que sea necesario o que cese en cualquier momento en que sea oportuno proceder al alta del paciente.

Por otro lado, otro precepto que invita a un desarrollo a la hora de su aplicación práctica, es el artículo 87, referido al deber de colaborar con las autoridades sanitarias cuando sea necesario para la efectividad de las medidas adoptadas, obligación que corresponde a todas las administraciones públicas. Otras administraciones han establecido protocolos mediante acuerdos entre las distintas la administraciones, estatal, autonómico y local para así concretar las funciones de Policía Local, Policía Nacional, Guardia Civil, Trabajadores Sociales y demás autoridades y colectivos.

En lo tocante a la cuestión de los recursos en vía administrativa, la ley de Gobierno Valenciano (9) establece en su artículo 68.3 c) que corresponde a los Secretarios Autonómicos rresolver los recursos que se interpongan contra las resoluciones de los centros directivos que estén bajo su dependencia y cuyos actos no agoten la vía administrativa, siendo que, según el Reglamento Orgánico y Funcional de la Conselleria de Sanidad Universal y Salud Pública (10), la Dirección General de Salud Pública, órgano competente en materia de control epidemiológico, depende de la Secretaría Autonómica de Salud Pública y del Sistema Sanitario Público, lo que conlleva que los actos de esta Dirección General son revisados en vía administrativa por el titular de la referida Secretaría Autonómica.

\section{LA EXPERIENCIA DEL REINO UNIDO}

Complementa este estudio el examen del derecho comparado aludiendo a la regulación de esta materia por otros países de derecho anglosajón; así, como ejemplo de estos nos referiremos a la normativa del Reino Unido, sin detenernos en ningún análisis exhaustivo

La Public Health (Control of Disesase) Act of 1984 (11) establece dos posibilidades de internamiento forzoso, en primer lugar el caso de pacientes de determinadas enfermedades infecciosas de los que se prevea que podrían no tomar las suficientes precauciones para evitar la propagación de la enfermedad y en segundo lugar, el caso de que los pacientes que al ser dados de alta de un centro sanitario no dispusieran de un alojamiento que fuera apropiado al efecto de tomar las 
precauciones necesarias para evitar dicha propagación. En estos casos se podría decretar su internamiento o su permanencia en el hospital apropiado, aunque ésta no fuera necesaria para los cuidados sanitarios del paciente.

Así, en su literalidad, la citada norma establece (Sections 37 y 38 de la Public Health Act of 1984):

\section{Removal to Hospital of Person with Notifiable Disease}

«37.- Where a justice of the peace (acting, if he deems it necessary, ex parte) is satisfied, on the application of the local authority, that a person is suffering from a notifiable disease and that his circumstances are such that proper precautions to prevent the spread of infection cannot be taken, or such precautions are not being taken, and that serious risk of infection is thereby caused to other persons, and that accommodation for him is available in a suitable hospital vested in the Secretary of state, or, pursuant to arrangements made by a Health Authority or Primary Care Trust (whether under an NHS contract or otherwise), in a suitable hospital vested in a NHS trust, Primary Care Trust or other person the justice may, with the consent mentioned in subsection $(1 \mathrm{~A})$ below, order him to be removed to it.

(1A) The consent referred to in subsection (1) above is that of a Primary Care Trust or Health Authority

(a) any part of whose area falls within that of the local authority, and

(b) which appears to the local authority to be an appropriate Primary Care Trust or Health Authority from whom to obtain consent.»

\section{Detention in Hospital of Person with Notifiable Disease.}

«38.-(1) Where a justice of the peace (acting, if he deems it necessary, ex parte) in and for the place in which a hospital for infectious diseases is situated is satisfied on the application of any local authority, that an inmate of the hospital who is suffering from a notifiable disease would not on leaving the hospital be provided with lodging or accommodation in which proper precautions could be taken to prevent the spread of the disease by him, the justice may order him to be detained in the hospital.

(2) An order made under Subsection (1) above may direct detention for a period specified in the order, but any justice of the peace acting in and for the same place may extend a period so specified as often as it appears to him to be necessary to do so.»

Siguiendo con la sistemática iniciada en este trabajo con arreglo a la cual nos preguntaríamos sobre la adecuación de la norma a los derechos fundamentales hemos de referirnos a su adecuación a la Human Rights Act, promulgada en 1998 trasponiendo el Convenio Europeo para la Protección de los Derechos Humanos y de las Libertades Fundamentales (12) al Derecho Británico

Así, el artículo 5 Convenio Europeo, cuyo párrafo 1.e) contempla el caso del internamiento, tiene la siguiente redacción:

Derecho a la libertad y a la seguridad.

1. Toda persona tiene derecho a la libertad y a la seguridad. Nadie puede ser privado de su libertad, salvo en los casos siguientes y con arreglo al procedimiento establecido por la ley: 
a. Si ha sido penado legalmente en virtud de una sentencia dictada por un tribunal competente.

b. Si ha sido detenido preventivamente o internado, conforme a derecho, por desobediencia a una orden judicial o para asegurar el cumplimiento de una obligación establecida por la ley.

c. Si ha sido detenido preventivamente o internado, conforme a derecho, para hacerle comparecer ante la autoridad judicial competente, cuando existan indicios racionales de que ha cometido una infracción o cuando se estime necesario para impedirle que cometa una infracción o que huya después de haberla cometido.

d. Si se trata del internamiento de un menor en virtud de una orden legalmente acordada con el fin de vigilar su educación o de su detención, conforme a derecho, con el fin de hacerle comparecer ante la autoridad competente.

e. Si se trata del internamiento, conforme a derecho, de una persona susceptible de propagar una enfermedad contagiosa, de un enajenado, de un alcohólico, de un toxicómano o de un vagabundo.

f. Si se trata de la detención preventiva o del internamiento, conforme a derecho, de una persona para impedir que entre ilegalmente en el territorio o contra la que este en curso un procedimiento de expulsión o extradición.

7. Toda persona detenida preventivamente debe ser informada, en el más breve plazo y en una lengua que comprenda, de los motivos de su detención y de cualquier acusación formulada contra ella.

8. Toda persona detenida preventivamente o internada en las condiciones previstas en el párrafo 1, c), del presente artículo deberá ser conducida sin dilación a presencia de un Juez o de otra autoridad habilitada por la ley para ejercer poderes judiciales, y tendrá derecho a ser juzgada en un plazo razonable o a ser puesta en libertad durante el procedimiento. La puesta en libertad puede ser condicionada a una garantía que asegure la comparecencia del interesado en juicio.

9. Toda persona privada de su libertad mediante detención preventiva o internamiento tendrá derecho a presentar un recurso ante un órgano judicial, a fin de que se pronuncie en breve plazo sobre la legalidad de su privación de libertad y ordene su puesta en libertad si fuera ilegal.

10. Toda persona víctima de una detención preventiva o de un internamiento en condiciones contrarias a las disposiciones de este articulo tendrá derecho a una reparación.»

Por su parte, el artículo 6 del mismo texto establece que:

«1. Toda persona tiene derecho a que su causa sea oída equitativa, públicamente y dentro de un plazo razonable, por un Tribunal independiente e imparcial, establecido por la Ley, que decidirá los litigios sobre sus derechos y obligaciones de carácter civil o sobre el fundamento de cualquier acusación en materia penal dirigida contra ella. La sentencia debe ser pronunciada públicamente, pero el acceso a la Sala de Audiencia puede ser prohibido a la prensa y al público durante la totalidad o parte del proceso en interés de la moralidad, del orden público o de la seguridad nacional en una sociedad democrática, cuando los intereses de los menores o la protección de la vida privada de las partes en el proceso así lo exijan o en la medida considerada necesaria por el Tribunal, cuando en circunstancias especiales la publicidad pudiera ser perjudicial para los intereses de la justicia.

2. Toda persona acusada de una infracción se presume inocente hasta que su culpabilidad haya sido legalmente declarada.

3. Todo acusado tiene, como mínimo, los siguientes derechos: 
a. A ser informado, en el más breve plazo, en una lengua que comprenda y detalladamente, de la naturaleza y de la causa de la acusación formulada contra él.

b. A disponer del tiempo y de las facilidades necesarias para la preparación de su defensa.

c. A defenderse por sí mismo o a ser asistido por un defensor de su elección y, si no tiene medios para pagarlo, poder ser asistido gratuitamente por un Abogado de oficio, cuando los intereses de la justicia lo exijan.

d. A interrogar o hacer interrogar a los testigos que declaren contra él y a obtener la citación y el interrogatorio de los testigos que declaren en su favor en las mismas condiciones que los testigos que lo hagan en su contra.

e. A ser asistido gratuitamente de un intérprete, si no comprende o no habla la lengua empleada en la Audiencia.»

La aplicación de los preceptos arriba reflejados ha sido criticada desde el punto de vista ético, así como en lo que respecta a su eficacia (véase el trabajo de Richard J. Coker, «The law, human rights, and the detention o individuals with tuberculosis in England and Wales") (13) Dichas críticas versan sobre la desproporción entre la conculcación de las libertades civiles y los beneficios obtenidos para la salud pública. También por la arbitrariedad en la duración de la limitación de la libertad del paciente y su falta de proporcionalidad con la persistencia del peligro para la salud pública.

Por otro lado, procedimentalmente se detectan deficiencias como el hecho de no tener tiempo, en el transcurso del procedimiento, a preparar una defensa adecuada ni existir el derecho a un defensor de oficio.

Además, en la práctica habría una falta de adecuación al Convenio Europeo para la Protección de los Derechos Humanos y de las Libertades Fundamentales tanto en lo que se refiere al control periódico del internamiento, así como en la falta de controles para asegurar que el internamiento se deba a una percepción cierta del riesgo.

En este sentido se proponen mayores salvaguardias de los derechos del individuo, como existían en la normativa anterior a 1968: un procedimiento imparcial y una defensa adecuada; también una revisión automática del proceso y una valoración continuada del riesgo en lugar de una valoración especulativa de un comportamiento futuro, con lo que la libertad de los individuos dejarían de limitarse automáticamente por períodos de tiempo innecesariamente largos.

Todo esto hace, además, que la actuación de los poderes públicos sea susceptible de revisión judicial y susceptible de dar lugar a reclamaciones de indemnizaciones por daños y perjuicios ( $A$. Harris y R. Martin, "The exercise of public health powers $i$ an era of human rights: the particular problems of tuberculosis») (14).

\section{CONCLUSIONES}

Nuestro derecho positivo admite la medida consistente en el aislamiento forzoso de una persona que padezca una enfermedad transmisible; esto supone la limitación de su Derecho a la Libertad Personal que es un Derecho Fundamental. Existen normas administrativas que prevén dicha medida que, en todo caso, deberá contemplar las garantías constitucionales en la limitación de tal Derecho Fundamental.

Con estos presupuestos hemos llegado a la conclusión de que se pueden compatibilizar el derecho constitucional a la Libertad Personal y el derecho a la protección de la salud pública.

Es de señalar que en la Comunidad Valenciana no existe una regulación procedimental exhaustiva en relación con la aplicación de las mencionadas medidas administrativas. 
No obstante, en todo momento hablamos de una herramienta que tiene un aspecto jurídico y otro clínico, de tal modo que su mayor o menor acierto técnico y adecuación jurídica no implica que clínicamente sea la medida más apropiada, circunstancia que no hemos entrado a valorar en este trabajo.

Hemos finalizado por examinar, como institución de derecho comparado, el caso de la normativa del Reino Unido, así como algunos comentarios críticos al respecto resultando que la misma sí es deficitaria de respeto por los derechos fundamentales de la persona.

\section{BIBLIOGRAFÍA}

1. Ley Orgánica 4/1981, de 1 de junio, de los estados de alarma, excepción y sitio. Boletín Oficial del Estado, n 134, (05-06-1981).

2. Ley Orgánica $3 / 1986$ de 14 de abril, de medidas especiales en materia de salud pública. Boletín Oficial del Estado, no 102, (29-04-1986).

3. Diario de Sesiones del Congreso de los Diputados núm. 249, sesión de 12/11/1985.

4. Garcia de Enterría E, Fernández TR. Curso de Derecho Administrativo. 17ª edición. En: Garcia de Enterría E, Fernández TR. Capítulo XIV, La coacción administrativa. Madrid, España: Civitas Ediciones; 2015. p. 763 a 808

5. Ley $41 / 2002$, de 14 noviembre de 2002 , básica reguladora de la autonomía del paciente y de derechos y obligaciones en materia de información y documentación clínica. Boletín Oficial del Estado n ${ }^{\circ}$ 274, (14-11-2002).

6. Sentencia 48/2001, de 13 de noviembre, del Tribunal Superior de Justicia de la Comunidad de

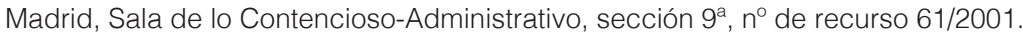

7. Ley 10/2014, de 29 de diciembre, de la Generalitat Valenciana, de Salud de la Comunitat Valenciana. Diario Oficial de la Comunidad Valenciana n 7434, (21-12-2014).

8. Ley 4/2005, de 17 de junio, de la Generalitat Valenciana, de Salud Pública de la Comunidad Valenciana. Diario Oficial de la Comunidad Valenciana, n 5034, (26-06-2005).

9. Ley 5/1983, de 30 de diciembre, de la Generalitat Valenciana, de Gobierno Valenciano. Diario Oficial de la Comunidad Valenciana $n^{\circ}$. 34, (09/02/1984).

10. Decreto 156/2015, de 18 de septiembre, del Consell, por el que aprueba el Reglamento orgánico y funcional de la Conselleria de Sanidad Universal y Salud Pública. Diario Oficial de la Comunidad Valenciana ${ }^{\circ}$ 7620, (22-09-205)

11. Public Health (Control of Disesase) Act of 1984 [monograph on the Internet]. London, United Kingdom: The National Archives; 1984 [cited feb 12, 2017]. Available from:

12. http://www.legislation.gov.uk/ukpga/1984/22/contents

13. Convenio Europeo para la Protección de los Derechos Humanos y de las Libertades Fundamentales, hecho en Roma el 4 de noviembre de 1950. Boletín Oficial del Estado, n² 243, (1010-1979)

14. Coker RJ. The law, human rights, and the detention of individuals with tuberculosis in England and Wales. J Public Health Med. 2000;22(3):263-7. PMID: 11077895

15. Harris A, Martin R. The exercise of public health powers in an era of human rights: the particular problems of tuberculosis. Public Health. 2004;118(5):313-22. DOI: 10.1016/j.puhe.2003.09.006, PMID: 15178137 